\title{
Einwirkungen des kalten Winters 1962/63 auf die Phytoplanktonentwicklung bei Helgoland
}

\author{
Max Gillbricht \\ Biologische Anstalt Helgoland, Zentrale, Hamburg-Altona
}

\begin{abstract}
Effects of the cold winter $1962 / 63$ on the phytoplankton development at Helgoland. During the cold winter 1962/63 the water temperature near Helgoland was extremely low (Fig. 1), and the minimum value was reached earlier in this year than it was in 1962. Because the plankton bloom began with stabilization of the water column after the minimum temperature was reached (better light conditions), phytoplankton development started earlier in 1963 than it did in 1962 (Fig. 8). This earlier start is also indicated by the amounts of the nutrients nitrogen and phosphate found in the water (Figs. 2, 4, 5, 6, 7). It is assumed that the phytoplankton bloom is stopped by grazing zooplankton later in the year (Fig. 10). Soluble organic substances (Fig. 11) must come from the sea bottom during the coldest period. This decomposition was greater in the cold winter of 1963 than in the year before. However, the phytoplankton development in this region as a function of time is difficult to assess because of the complicated hydrographical situation, involving different bodies of water, different salinities, etc.
\end{abstract}

\section{EINLEITUNG}

Es soll hier ein erster Überblick über die im Zusammenhang mit dem kalten Winter 1962/63 beim Phytoplankton und in seiner Umwelt zu beobachtenden Besonderheiten gegeben werden. Leider liegen keine entsprechenden mehrjährigen Beobachtungen zur Bestimmung des Normalverlaufs vor, da wir erst im Januar 1962 mit diesen Untersuchungen begonnen haben. Wir können den Eiswinter daher nur mit dem vorhergehenden Jahr vergleichen.

\section{UNTERSUCHUNGSERGEBNISSE}

Wie Abbildung 1 zeigt, waren die Oberflächentemperaturen (Dekadenmittel werktäglicher Beobachtungen) auf der Reede von Helgoland im Februar 1963 sehr niedrig. Im Jahre 1962 trat das Temperaturminimum später auf und erreichte nicht diesen Extremwert. Bezeichnend ist, daß auch die Sommertemperaturen 1963 abnorm waren. Wir hatten es also im Berichtsjahr im ganzen mit kontinentaleren Verhältnissen zu tun. Der Salzgehalt (anfangs yom Deutschen Hydrographischen Institut mit einem Salinometer, von der dritten Oktoberdekade 1962 ab mit dem Aräometer be- 
stimmt) weist, wie bekannt (GOEDECKE 1964), starke unregelmäßige Schwankungen auf mit einem schwach angedeuteten Minimum im Sommer und ist in beiden Jahren sehr verschieden. Die ständigen Anderungen lassen darauf schließen, daß wir stets ein anderes Wasser mit einer anderen Bevölkerung untersuchen, und daß daher die zu beobachtenden biologischen Unterschiede nicht nur eine Funktion der Zeit, sondern aud des Salzgehalts sind. Selbst wenn dieser etwa konstant bleibt, was im vorliegenden Falle allenfalls von Februar bis Mai 1963 (mit einer Ausnahme) gilt, dann haben

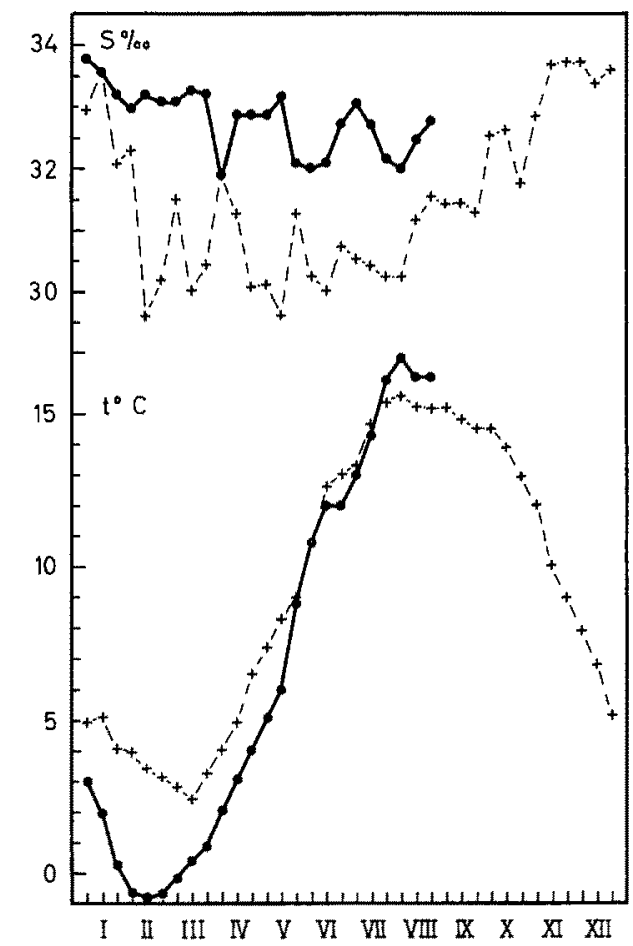

Abb. 1: Dekadenmittel der Oberflächenwerte Helgoland-Reede von Temperatur und Salzgehalt für $1962(+\ldots)$ und $1963(\bullet-)$

wir es de facto immer noch mit einem ständigen küstenparallelen Strom zu tun, in dem die Organismen zwar längere Zeit unter ähnlichen Bedingungen leben, aber die einzelnen Proben aus verschiedenem Wasser stammen. Es ist daher auch bei konstantem Salzgehalt nur bedingt möglich, Aussagen über zeitliche Veränderungen zu machen.

Betrachten wir unter diesen Gesichtspunkten einmal die Kurven des Nitratstickstoffs (Abb. 2, $\mu$ g N/1; Bestimmung nach Strickland \& Parsons 1960; Dekadenmittel, Beobachtung an jedem zweiten Werktag, dies gilt auch für die folgenden Bestimmungen). 1962 erreicht das Nitrat im Frühjahr sein Maximum, um dann bis zum Juli zu geringen Werten abzufallen und bei dieser Menge bis zum Jahresende zu bleiben. Das kommende Frühjahr bringt einen geringen Anstieg und von April ab wiederum einen Abfall, der also früher als im Vorjahre beginnt. Das Bild ist so aber 


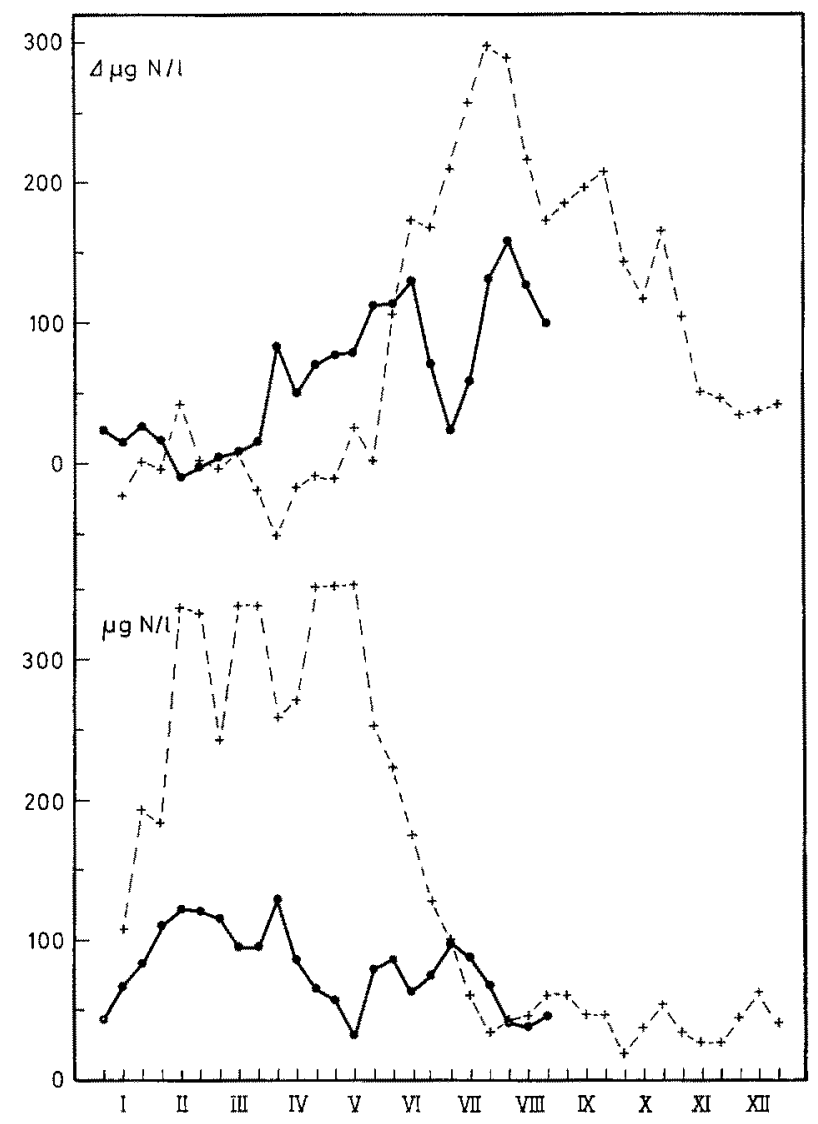

Abb. 2: Jahreskurven des Nitratstickstoffs $(\mu \mathrm{g} N / 1)$ und des berechneten Nitratverbrauchs $(\triangle \mu \mathrm{g} \mathrm{N} / \mathrm{l})$ für $1962(+\cdots)$ und $1963(\bullet-)$

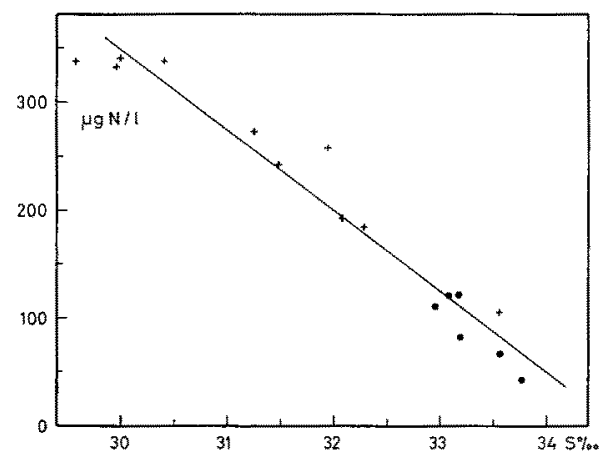

Abb. 3: Beziehung zwischen Salzgehalt und Nitratmenge in den produktionsarmen Wintermonaten. 1962: Januar bis 20. April; 1963: Januar bis 28. Februar. 1962: +; 1963: • 


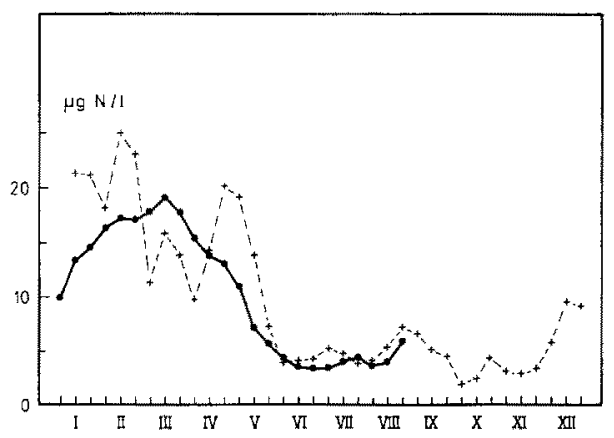

Abb. 4: Jahreskurven des Nitritstickstoffs für $1962(+\ldots \ldots)$ und 1963

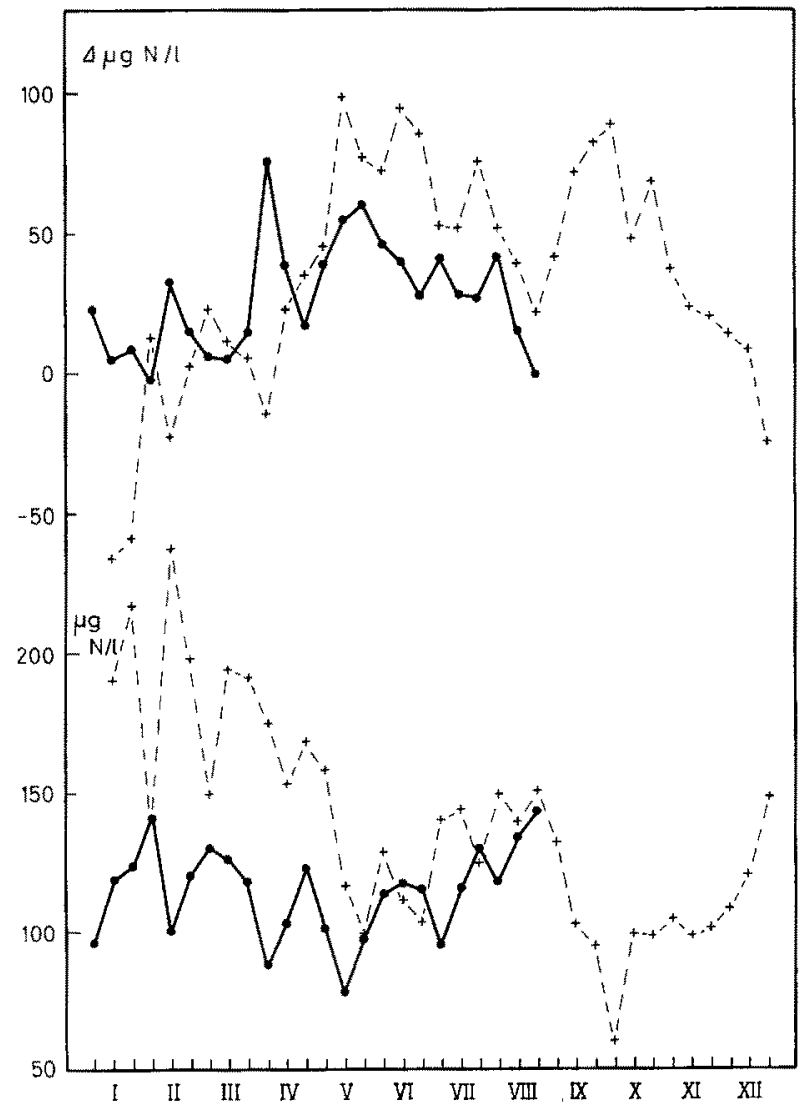

Abb. 5: Jahreskurven des Ammoniakstickstoffs $(\mu \mathrm{g}$ N/l) und des berechneten Ammoniakverbrauchs $(\triangle \mu \mathrm{g} N / 1)$ für $1962(+---)$ und $1963(\bullet-)$ 
recht unklar. Wo bleiben z. B. die anfangs vorhandenen Nitratmengen? Um dies zu klären, wurde der Zusammenhang zwischen Salzgehalt und Nitrat vor Beginn der Frühjahrswucherung bestimmt (Abb. 3). Es läßßt sich dann zeigen, daß3 das Nitrat mit dem Süßwasser eingespült wird. Der Korrelationskoeffizient zwischen Nitrat und Salzgehalt ist mit $\mathrm{r}=-0,98$ bei $\mathrm{n}=16$ sehr hoch. Es dürfte daher interessant sein, einmal zu ermitteln, welche Differenz zwischen dem aus dem Salzgehalt berechneten und dem beobachteten Nitratgehalt besteht ( $\triangle \mu \mathrm{g} N / 1$ in Abb. 2). Es wird also angezeigt, wie groß das Nitratdefizit jeweils ist. Bei der Unsicherheit solcher Berechnungen ist es weiter nicht verwunderlich, daß sich gelegentlich auch negative Werte beobachten

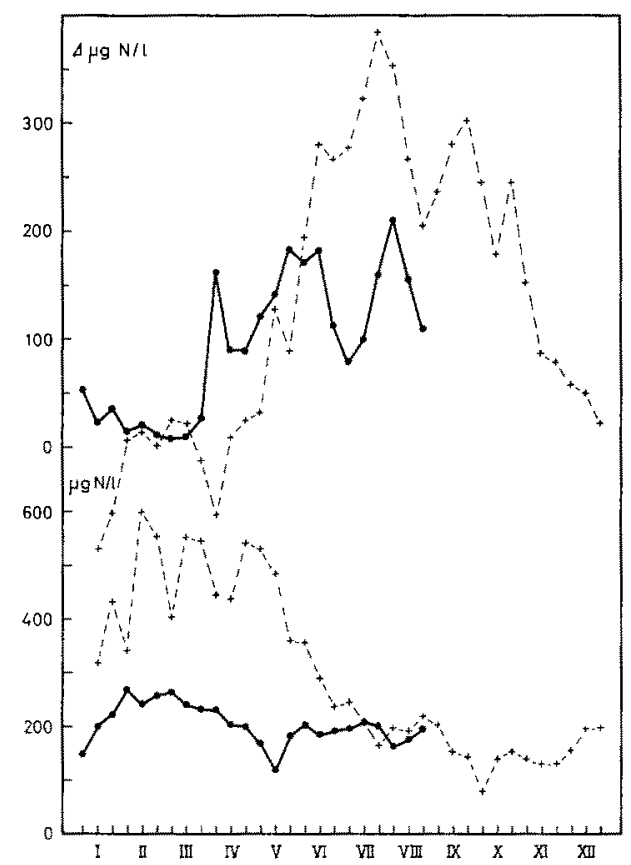

Abb. 6: Jahreskurven des anorganischen Stickstoffs ( $\mu \mathrm{g} \mathrm{N/l)}$ und des berechneten Verbrauchs $(\triangle \mu \mathrm{g} N / 1)$ für $1962(+---)$ und $1963(\bullet-)$

lassen. Sehr gut kommt dabei aber das biologische Geschehen zum Ausdruck. So lassen sich im Juli, September und Oktober 1962 Maxima der Nitratzehrung beobachten, die sich auch in der Phytoplanktonkurve (Abb. 8) wiederfinden. Zum Herbst wird durch Remineralisationsvorgänge das Nitrat wieder in Freiheit gesetzt. 1963 war der Nitratverbrauch erheblich kleiner und die Bindung zum Phytoplankton geringer als im Vorjahr. Der Grund zur verminderten Aufnahme liegt vielleicht darin, daß auch das Angebot kleiner war. Wichtig aber ist der zeitige Beginn der Zehrung im Jahre 1963.

Das Nitrit (Abb. 4; Bestimmung nach Strickland \& Parsons 1960) als instabi-

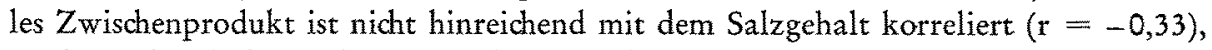
um besondere Zehrungskurven zu bringen, die praktisch spiegelbildlich zur vorliegenden Darstellung verlaufen. Man hat den Eindruck, daß hier nur die Aktivität der ver- 
schiedenen Stickstoffoxydierer zum Ausdruck kommt. Im Sommer geht die Nitrit- und im Winter die Ammoniakoxydation besonders schnell vor sich. Es ist möglich, daß bei den tiefen Temperaturen im Frühjahr 1963 zunächst sogar die Nitritbildung weitgehend blockiert wurde, so daß das Maximum später und weniger ausgeprägt eintrat.

Genauere Aussagen erlaubt der Ammoniakstickstoff (Abb. 5; Bestimmung nach GILlbRICHT 1961a). Die direkte Auftragung der Meßwerte ( $\mu \mathrm{g} N / 1)$ sagt zwar nicht viel aus, aber die Bindung an den Salzgehalt ist auch bei dieser nicht sonderlich stabilen Substanz vor Beginn der Frühjahrswucherung noch beachtlich hoch $(\mathrm{r}=-0,75)$, so

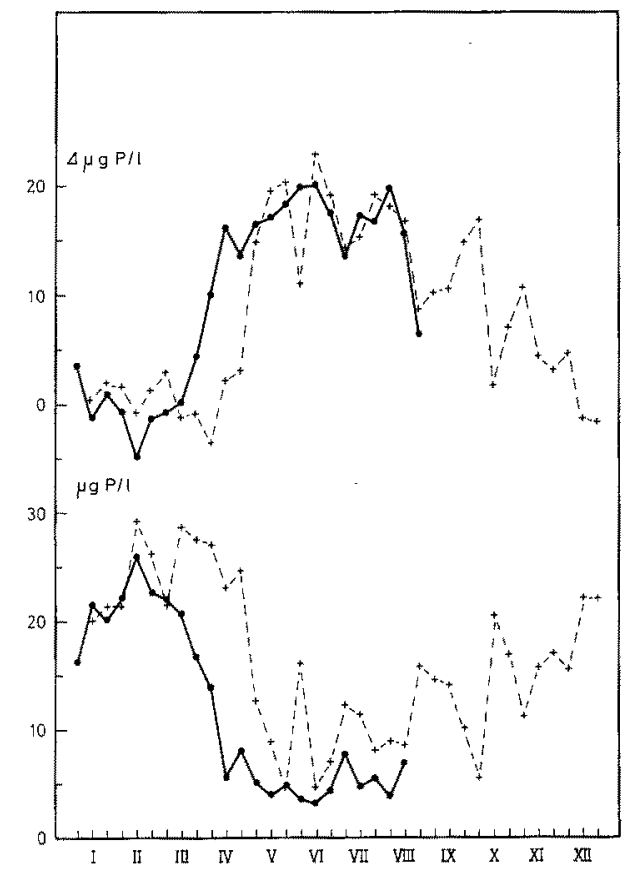

Abb. 7: Jahreskurven des Phosphats $(\mu \mathrm{g} \mathrm{P} / 1)$ und des berechneten Phosphatverbrauchs $(\triangle \mu \mathrm{g} \mathrm{P} / \mathrm{l})$ für $1962(+\cdots)$ und 1963

daß sich wiederum das Defizit ( $\triangle \mu \mathrm{g} N / 1$ ) bestimmen läßt. Zehrung im Frühjahr (1963 früher als 1962) und Remineralisation im Herbst kommen so gut zum Ausdruck. Bis $\mathrm{zu}$ einem gewissen Grade besteht auch eine Beziehung zu den einzelnen Planktonmaxima (Abb. 8).

Bei der Summe der anorganischen Stickstoffverbindungen (Abb. 6) läßt sich ebenfalls das Defizit ( $\triangle \mu \mathrm{g} N / 1)$ nach Eliminierung des Salzgehalts bestimmen $(\mathrm{r}=-0,96)$. Der Zusammenhang mit dem Phytoplankton (Abb. 8) ist 1962 besser als 1963. Es ist wiederum gut zu erkennen, daß die Zehrung im letzten Beobachtungsjahr früher beginnt als im vorhergehenden. In diesem Fall interessieren aber vor allem die direkten Meßwerte $(\mu \mathrm{g} \mathrm{N} / 1)$. Es ist - vorzugsweise in der Form von Ammoniak - immer so viel gebundener anorganischer Stickstoff yorhanden, daß $\mathrm{N}$ während der Beobachtungszeit kaum Minimumfaktor gewesen sein dürfte. Allerdings spricht die Ammoniakbestim- 
mung auch auf einige organische Verbindungen an, so daß die Werte vielleicht zu hoch liegen. Jedoch nimmt nach Schreiber (1927) z. B. Carteria auch Glykokoll als N-Quelle.

Das Phosphat (Abb. 7; Bestimmung modifiziert nach Kalle 1934) ist im Frühjahr ebenfalls eng mit dem Salzgehalt korreliert $(r=-0,78)$; jedoch ergibt schon die direkte Eintragung der Meßwerte ( $\mu \mathrm{g} \mathrm{P} / \mathrm{l})$ die typischen Jahreskurven. Der Phosphatverbrauch $(\triangle \mu \mathrm{g} P / 1)$ war in beiden Jahren - wie das Angebot - etwa gleich, aber es ließ sich auch in diesem Falle 1963 ein früherer Beginn der Zehrung beobachten als 1962. Phosphat dürfte kein Minimumfaktor gewesen sein.

Vergleichen wir diese Befunde mit der bei Helgoland durchgeführten experimentell-biologischen Nährstoffuntersuchung von SCHREIBER (1927), so ergeben sich man-

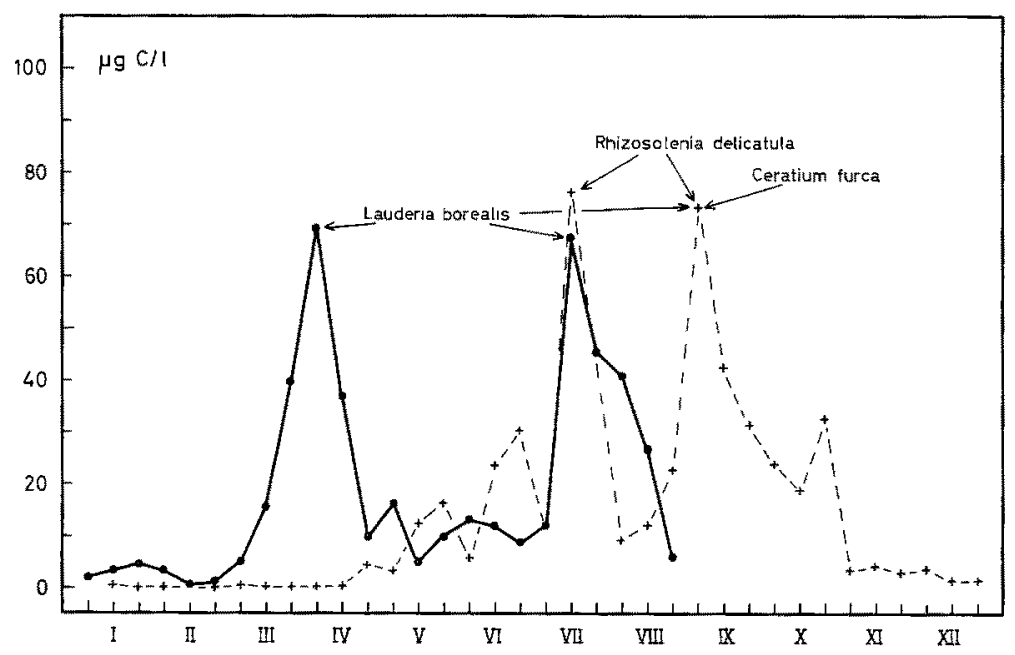

Abb. 8: Jahreskurven des Phytoplanktons für $1962(+\ldots)$ und $1963(\bullet-)$

cherlei Unterschiede. Seine Phosphatwerte dürften zu hoch sein, auch wenn während seiner Beobachtungen (August 1926 bis Juli 1927) der Salzgehalt relativ gering war (Gokdecke 1956). Vermutlich haben die Organismen (Carteria) beim Test zuviel P geboten bekommen und entsprechend zuviel aufgenommen. Der $\mathrm{N}$-Wert ist mit unseren Befunden vergleichbar. Im Sommer wurde Nährstoffmangel beobachtet. $\mathrm{Ob}$ sich die Verhältnisse geändert haben, ob sie in den einzelnen Jahren verschieden sind oder ob chemische und physiologische Methoden eben unterschiedliche Ergebnisse liefern, ist schwer zu sagen.

Es ist ferner noch von Interesse, daß sich aus den von KüHL \& MANN (1958) gebrachten Diagrammen am 16., 18. und 23. November 1956 eine andere Beziehung zwischen Nitrat und Salzgehalt ergab, als 1962 und 1963 bei Helgoland beobachtet wurde. Der Schnittpunkt der beiden Geraden liegt bei einem Salzgehalt, der 1956 etwa den Verhältnissen bei Feuerschiff Elbe I entsprach. In dieser Gegend zeigte auch das t/S-Diagramm bei einer eigenen Untersuchung (16./17. März 1960) einen Knick. Es handelt sich hierbei also um die z. B. auch yon der Jade (GILlBricht 1956) be- 
kannte Tatsache, daß sich das Wasser der Flüsse und tiefen Buchten nicht direkt mit dem Wasser der Deutschen Bucht vermischt, sondern daß hier das Wattenmeerwasser als besonderer Körper eingeschoben ist.

Die Jahreskurven des Phytoplanktons (Abb. 8; Bestimmung nach Gillbricht 1959, 1962a) bestätigen die Beobachtungen bei den Nährstoffkurven: 1963 begann die Wucherung mehrere Monate früher als 1962. Wie ist dieser Unterschied zu erklären? 1963 wurden ungewöhnlich tiefe Temperaturen beobachtet (Abb. 1), entsprechend früher setzte dann die Erwärmung des Wassers und damit eine gewisse Stabilisierung ein. Als Folge hiervon nimmt die Trübung des Wassers ab (GILlbricht 1962b), das

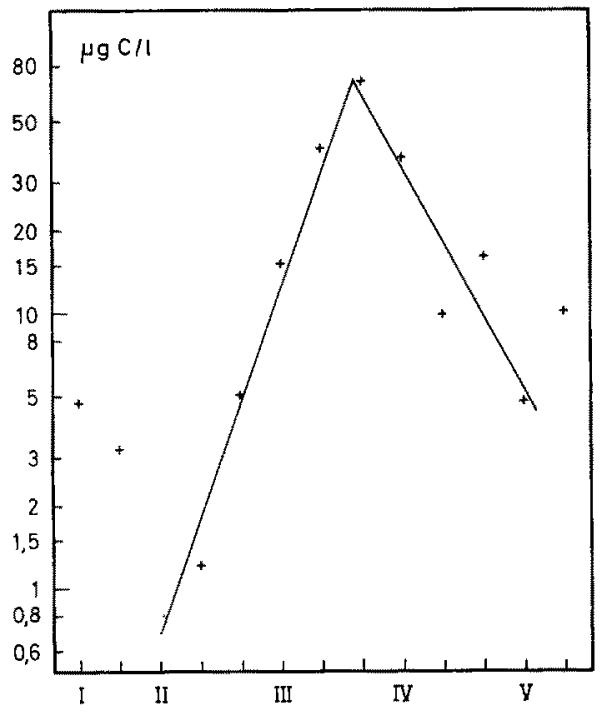

Abb. 9: Frühjahrsmaximum des Phytoplanktons 1963

Phytoplankton erhält jetzt genügend Licht, und die Wucherung beginnt. Haben wir es immer mit etwa demselben Wasserkörper zu tun (wie im Frühjahr 1963; Abb. 9), so erfolgen Wachstum (tägliche Zunahme 10\%) und weniger deutlich auch Abfall (tägliche Abnahme 6\%) logarithmisch. Der Maximalwert wurde bei einem abweichenden (niedrigeren) Salzgehalt beobachtet. Sein Hineinpassen in die übrigen Werte ist also mehr oder weniger zufällig. 1962 begann die Wucherung erst einen Monat nach dem Temperaturminimum (Abb. 8). Dieser Umstand ist wohl so zu erklären, daß 1962 das Temperaturminimum mit einem Salzgehaltsminimum zusammenfiel (Abb. 1). Nun bedeutet aber höherer Salzgehalt einen späteren Eintritt des Minimums bei einer höheren Temperatur. Es dürfte also längere Zeit in Wasser gemessen worden sein, welches jeweils das Minimum allenfalls gerade überschritten hatte, und nur bei Einsetzen einer echten Erwärmung ist ja mit dem Beginn der Planktonwucherung zu rechnen. Der folgende unruhige Verlauf der Planktonblüte ist sicher wenigstens zum Teil auf den an Hand der Salzgehaltsfluktuationen zu beobachtenden, ständigen Wasserwechsel zurïckzuführen.

Es ist überraschend, daß die Hauptart (die Diatomee Lauderia borealis) vom 
Sommer bis zum Herbst und in den verschiedenen Jahren dominiert. Ceratium furca hingegen scheint erst spät im Jahr in größeren Mengen aufzutreten. Frühere Beobachter (z. B. Braarud, GaArder \& Grøntved 1953) haben jedoch andere Diatomeen als Massenformen beobachtet, so daß auch mit langfristigen Anderungen oder unperiodischen Schwankungen zu rechnen ist.

Nun sind zwar der Beginn der Phytoplanktonentwidklung im Frühjahr und entsprechend das Ende im Herbst recht einfach zu erklären, aber die Vorgänge während des Sommers sind erheblich schwerer zu deuten. Für das plötzliche Abbrechen einer Wucherung kommen drei Gründe in Betracht.

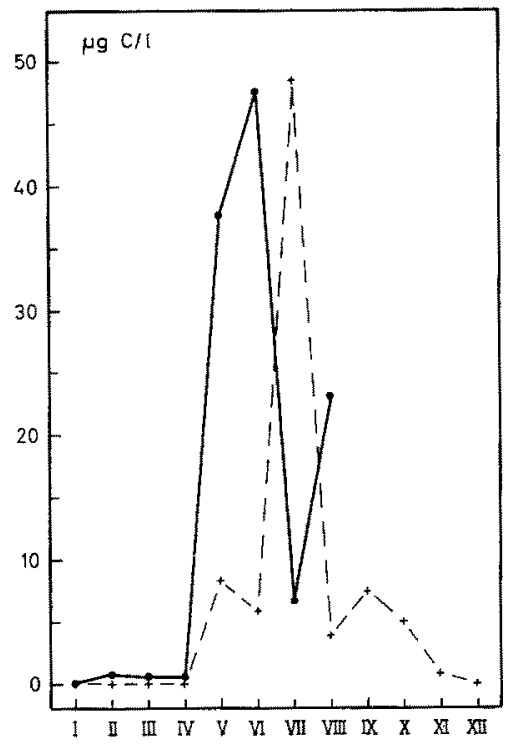

Abb. 10: Jahreskurven des Zooplankrons für $1962(+---)$ und $1963(-\bullet)$

1. Die Turbulenz nimmt plötzlich zu, etwa infolge eines Sturmes (z. B. GiLLBRICHT 1955). Nach den Vertikalserien in der Umgebung Helgolands lag dieser Tatbestand wohl nicht vor.

2. Die Nährstoffvorräte sind erschöpft (z. B. GILlBRICHT 1952). Dies dürfte nach den vorliegenden Untersuchungen wenigstens hinsichtlich $N$ und $P$ kaum der Fall gewesen sein.

3. Die Zehrung durch das Zooplankton vermindert das Phytoplankton. Nun streuen die eigenen Zooplanktonzählungen wegen der kleinen untersuchten Wassermengen und der Größe der meisten Organismen (z. B. werden schon Copepoden nur selten gefunden) so stark, daß nur Monatsmittel betrachtet werden können (Abb. 10). Aber es läßt sich auch so wahrscheinlich machen, daß ein Wechselspiel zwischen Phytound Zooplankton besteht, indem das Zooplankton etwa mit oder kurz nach dem Phytoplankton (das ist bei Monatsmitteln schwer zu sagen) sein Maximum erreicht. Das erste Zooplanktonmaximum 1963 liegt im Vergleich zum Phytoplankton zu spät. Es ist gut möglich, daß der kalte Winter die Zooplanktonentwicklung verzögert hat. 
Im Jahre 1958 hatte sich bei Grönland eine enge Beziehung zwischen der organischen Substanz (Bestimmung nach GiLlBRicht 1957) und dem Phytoplankton ergeben (GILLBRICHT 1961b). Untersucht man diesen Zusammenhang in unserem Gebiet (Abb. 11), so sieht es hier ganz anders aus. Das C-Maximum (wegen der starken Streuung wiederum nur Monatsmittel) liegt stets vor dem Phytoplanktonmaximum. Bestimmt man jetzt wie bei den Nährstoffen die Korrelation zwischen $\mathrm{C}$ und Salzgehalt im Frühjahr, so ergibt sich $r=+0,02$; es besteht also praktisch keine Bezie-

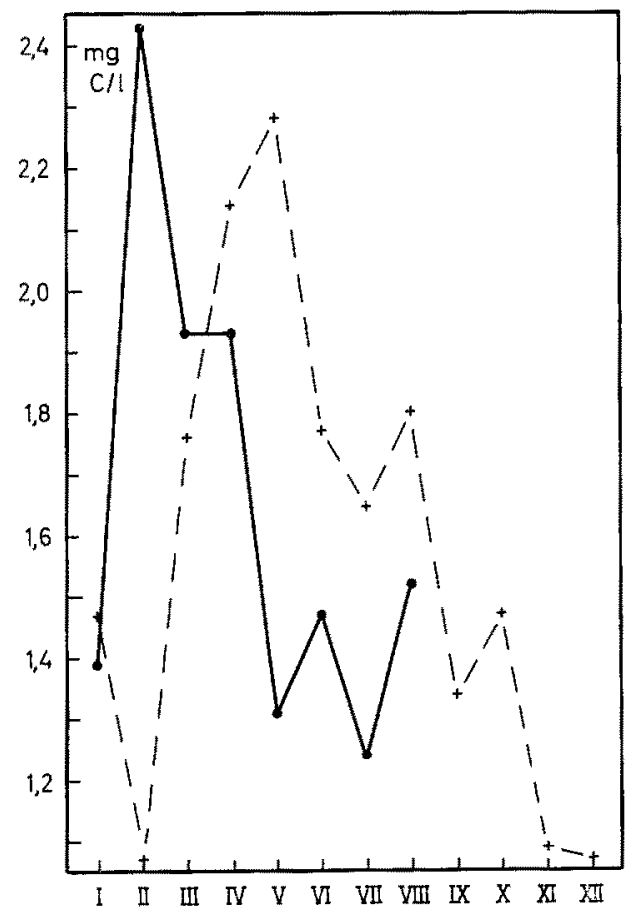

Abb. 11: Jahreskurven der oxydierbaren Substanz für $1962(+\ldots)$ und $1963(\bullet-)$

hung zwischen diesen beiden Größen. Nun kann dieses Ergebnis nicht generell richtig sein, denn Abb. 12 zeigt nicht nur, daß der pH-Wert (Bestimmung nach GillBricht 1953) jahreszeitlich stark schwankt und daß - wie wir schon wissen - das Phytoplankton 1963 früher zu wuchern begann als 1962, sondern es läßt sich vielmehr ganz grob durch Mittelung aller $\mathrm{pH}^{-}, \mathrm{t}^{0}$ - und $\mathrm{S}^{0} / 00$-Werte berechnen (Buch 1951), daß im Durchschnitt in jedem Liter Wasser etwa $1 / 3 \mathrm{mg} \mathrm{C}$ in Form der Kohlensäurekonstituenten mehr vorhanden ist, als dies im Falle eines Ausgleichs mit der Luftkohlensäure der Fall sein sollte. Es wird folglich mehr Kohlensäure gebildet als bei der Assimilation verbraucht wird, d. h. das Seegebiet ist insgesamt als heterotroph anzusehen und muß folglich organische Stoffe von außen zugeführt bekommen. Dies kann praktisch nur mit dem Süßwasser erfolgen. Tatsächlich ergibt sich nach dem Abklingen des C-Maximums im Frühjahr (Juli bis Dezember 1962 und Mai bis August 1963) eine Beziehung zwi- 
schen $\mathrm{C}$ und $\mathrm{S} \%$ von $\mathrm{r}=-0,44$. Entsprechend korrigierte Kurven sehen jedoch im Prinzip nicht anders aus als Abb. 11. Es ist aber zu beachten, daß so der Februarwert 1962 stark negativ wird. Die ersten Anzeichen hierfür lassen sich im Protokoll bereits im Anfang des Monats nachweisen, jedoch dürfte das Phänomen vorzugsweise mit der schweren Sturmflut zusammenhängen. GunkeL (1964) hat zu dieser Zeit auch abnorme Bakterienzahlen beobachtet. Es dürfte sich mithin um einen regionalen Vorgang gehandelt haben. Der betreffende Wasserballen ist mit dem Reststrom in kurzer Zeit aus dem Beobadhtungsgebiet verschwunden. Wir müssen diesen Wert daher bei der Beurteilung, welche zeitlichen Vorgänge sich wirklich im Wasser abgespielt haben, ver-

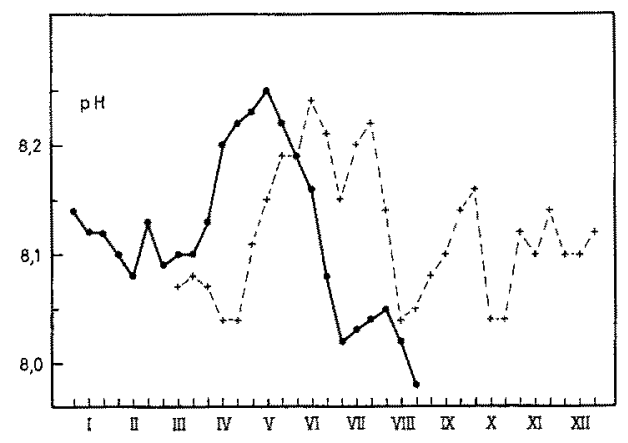

Abb. 12: Jahreskurven des pH-Wertes für $1962(+---)$ und $1963(\bullet-)$

werfen. Unter Berücksichtigung dieser Tatsache errechnet sich (nach Eliminierung des Salzgehaltseinflusses) im Frühjahr 1962 ein Anstieg der C-Menge um 0,5 mg C/1 und 1963 entsprechend um $1 \mathrm{mg} \mathrm{C/1}$. Nun ließ sich aber oben (S. 272) zeigen, daß zur Zeit dieses C-Maximums kein Zusammenhang zwischen $C$ und $S$ bestand. Die Substanzen müssen also an Ort und Stelle entstanden sein und mithin aus dem Boden stammen. Diese Abbauvorgänge beginnen offenbar etwa mit dem Temperaturminimum. Es handelt sich hierbei um ganz beträchtliche Mengen. Rechnen wir für unser Gebiet mit $20 \mathrm{~m}$ Wassertiefe, so bedeutet dies für $196210 \mathrm{~g} \mathrm{C} / \mathrm{m}^{2}$ und für $196320 \mathrm{~g} \mathrm{C} / \mathrm{m}^{2}$. Das ist ein beachtlicher Teil der jährlich zu erwartenden Phytoplanktonproduktion. Die Anreicherung von so viel organischer Substanz im Boden dürfte durch die Tatsache begünstigt werden, daß sich während der Planktonblüte viel Phytoplankton am Boden ansammelt (GrLbRICHT 1962b). Daß in strengen Wintern erheblich mehr Lebewesen im Boden sterben als sonst, deckt sich auch mit den Beobachtungen von Ziegelmeier (1964).

\section{ZUSAMMENFASSUNG}

1. Nach dem kalten Winter $1962 / 63$ setzten die Erwärmung des Wassers und damit auch die Phytoplanktonentwicklung bei Helgoland früher als im Vorjahre ein.

2. Dies zeigen ebenfalls die Nährstoffuntersuchungen $(N, P)$.

3. Gelöste organische Stoffe werden etwa zur Zeit des Temperaturminimums in großer 
Menge aus dem Boden abgegeben. Diese Absterbevorgänge dürtten im kalten Winter erheblich größer gewesen sein als im Jahr davor.

4. Die komplizierten hydrographischen Verhältnisse erschweren das Erkennen und Deuten der biologischen Vorgänge.

Mein besonderer Dank gilt im Zusammenhang mit den unter schwierigen Eisverhältnissen durchgeführten Winterbeobachtungen 1963 den Kapitänen und Besatzungen des Seenotrettungskreuzers "Hindenburg" der Deutschen Gesellschaft zur Rettung Schiffbrüchiger, des F. K. "Uthörn“ und des Motorboots „Ellenbogen“.

\section{ZITIERTE LITERATUR}

Braarud, T., Gaarder, K. \& Grontved, J, 1953. The phytoplankton of the North Sea and adjacent waters in May 1948. Rapp. Cons. Explor. Mer 133, 1-87.

BucH, K., 1951. Das Kohlensäure-Gleichgewichtssystem im Meerwasser. Havsforskninginstitutets Skrift. 151, 1-18.

Gillbricht, M., 1952. Untersuchungen zur Produktionsbiologie des Planktons in der Kieler Bucht. II. Kieler Meeresforsch. 9, 51-61.

- 1953. Kolorimetrische pH-Messung im Seewasser unter Verwendung von Kresolrot. Kurz. Mitt. Fisch. Biol. 2, 1-21.

- 1955. Wucherungen von Phytoplankton in einem abgeschlossenen Hafenbecken. Helgol. Wiss. Meeresunters. 5, 141-168.

- 1956. Die Hydrographie des Jadebusens und der Innenjade. Veröff. Inst. Meeresforsch. Bremerhaven 4, 153-170.

- 1957. Ein Verfahren zum oxydativen Nachweis von organischer Substanz ím Seewasser. Helgol. Wiss. Meeresunters. 6, 76-83.

- 1959. Das Phytoplankton im nördlichen Nordatlantischen Ozean im Spätwinter und Spätsommer 1958. Dtsch. bydrogr. Z. Erg. H. B 3, 90-93.

- 1961a. Eine Methode zum direkten Nachweis von Ammoniak im Seewasser. Helgol. Wiss. Meeresunters. 8, 57-67.

- 1961b. The distribution of microplankton and organic matter in the northern North Atlantic during late winter and late summer 1958. Rapp. Cons. Explor. Mer 149, 189-193.

- 1962a. Uber das Auszählen von Planktonschöpfproben. Helgol. Wiss. Meeresunters. 8, 203 bis 218.

- 1962b. Die Frühjahrswucherung des Phytoplanktons in einem flachen Gezeitenmeer. Kieler Meeresforsch. 18, 151-176.

Goedecke, E., 1956. Mean values of temperature and salinity derived from periodic observations made at Helgoland roads. Annls biol., Copenh. 11, 46-47.

- 1964. Zur Hydrographie der Deutschen Bucht während des Winters 1962/63. Helgol. Wiss. Meeresunters. 10, 231-245.

GunkEL, W., 1964. Einwirkungen des kalten Winters 1962/63 auf die Bakterienpopulation der Nordsee. Helgol. Wiss. Meeresunters. 10, 246-256.

Kalle, K., 1934. Meereskundlich-chemische Untersuchungen mit Hilfe des Zeiss'schen PulfrichPhotometers. III. Mitt. Methodische Untersuchungen der Phosphatgehalts-Bestimmung. Annln Hydrogr. Berl. 62, 65-74.

KüHL, H. \& MaNN, H., 1958. Das Verhalten anorganischer Stickstoffverbindungen im Mündungsgebiet eines Flusses. Arch. Fisch. Wiss. 9, 9-16.

Schreiber, E., 1927. Die Reinkultur von marinem Phytoplankton und deren Bedeutung für die Erforschung der Produktionsfähigkeit des Meerwassers. Wiss. Meeresunters. Abt. Helgoland N.F. 16, 10, 1-34.

Strickland, J. \& Parsons, T., 1960. A manual of sea water analysis. Bull. Fish. Res, Bd Can. $125,1-185$.

ZiegelmeIer, E., 1964. Einwirkungen des kalten Winters 1962/63 auf das Makrobenthos im Ostteil der Deutschen Bucht. Helgol. Wiss. Meeresunters. 10, 276-282. 


\section{Diskussion im Anschluß an den Vortrag GILLBRICHT}

KüHL: Als Ergänzung zu den Ausführungen von Herrn Dr. GiLlbricht: Bei PlanktonserienUntersuchungen vor Cuxhaven konnten wir eine Massenentwidklung von Biddulphia aurita den ganzen April 1963 über feststellen.

v. Stosch: Man möge bei dem Zusammenbrechen von Planktonvegetationen die Rolle der Parasiten nicht übersehen. Ich erinnere an das zweimal in der Nordsee (Norderney 1952, List 1953) beobachtete Verschwinden einer Streptotbeca-Population durch den offenbar für die Art recht spezifischen Peridineen-Parasiten Paulsenella innerhalb weniger Tage. Die jeweils überlebenden Zellen machten dabei einen gesunden Eindruck.

GILlBRrCHT: Ob Parasitenbefall vorlag, dürfte an jodfixiertem Material bei Massenbestimmungen schwer zu entscheiden sein. Meine Ansicht hinsichtlich des Zusammenhangs zwischen Phyto- und Zooplankton habe ich wohl zu scharf formuliert. Man kann korrekt nur sagen, daß die Beobachtung der Annahme nicht widerspricht, daß das Phytoplankton durch das Zooplankton erheblid dezimiert wird.

v. STOSCH: Ich hatte gegen Ende der Hochproduktion der Diatomeen (August/September 1952 und 1953 in List) den Eindruck, daß diese unter N, P oder Fe-Mangel litten, was am Habitus der Zellen (Farbe, Leukosingehalt) erkennbar ist, jedenfalls aber unter einem Nährstoffmangel. Das Aneignungsvermögen für die einzelnen Nährstoffe ist jedoch zweifellos artspezifisch.

GrLlbRicht: Natürlich müssen wir - zumindest bei einzelnen Arten - immer mit Mangelerscheinungen rechnen, selbst dann wenn chemisch noch Nährstoffe nachgewiesen werden können. Die Grenzkonzentrationen für die einzelnen Formen sind noch nicht bekannt. Außerdem können schließlich auch nichtbestimmte Spurenstoffe das Wachstum begrenzen. 